\title{
Successful robotic resection of a primary cardiac synovial sarcoma
}

Akbarshakh Akhmerov, MD, ${ }^{\mathrm{a}}$ Joshua S. Chung, MD, ${ }^{\mathrm{b}}$ Dominic Emerson, MD, ${ }^{\mathrm{a}}$ Joanna Chikwe, MD, ${ }^{\mathrm{a}}$ and Alfredo Trento, $\mathrm{MD}^{\mathrm{a}}$

\footnotetext{
From the a Department of Cardiac Surgery, Smidt Heart Institute, Cedars-Sinai Medical Center, Los Angeles, Calif; and ${ }^{\mathrm{b}}$ Department of Cardiothoracic Surgery, Loma Linda University Medical Center, Loma Linda, Calif. Dr Akhmerov is supported by an institutional training grant (T32HL116273).

Disclosures: The authors reported no conflicts of interest.

The Journal policy requires editors and reviewers to disclose conflicts of interest and to decline handling or reviewing manuscripts for which they may have a conflict of interest. The editors and reviewers of this article have no conflicts of interest.

Received for publication Dec 29, 2019; accepted for publication Feb 2, 2020; available ahead of print Feb 24, 2020.

Address for reprints: Alfredo Trento, MD, Department of Cardiac Surgery, Smidt Heart Institute, Cedars-Sinai Medical Center, 127 S San Vicente Blvd, Los Angeles, CA 90048 (E-mail: alfredo.trento@cshs.org).

JTCVS Techniques 2020;2:104-6

2666-2507

Copyright (C 2020 The Authors. Published by Elsevier Inc. on behalf of The American Association for Thoracic Surgery. This is an open access article under the CC BY-NC-ND license (http://creativecommons.org/licenses/bync-nd/4.0/).

https://doi.org/10.1016/j.xjtc.2020.02.005
}

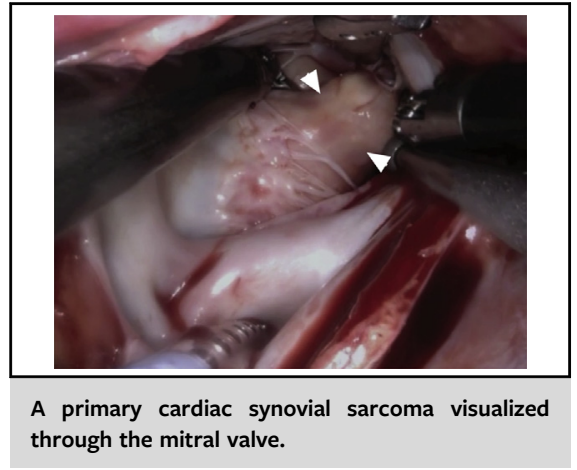

\begin{abstract}
CENTRAL MESSAGE
Primary cardiac synovial sarcomas are rare cardiac tumors and mandate surgical resection. This case report details a novel, robotic, minimally invasive excision of this rare neoplasm.
\end{abstract}

See Commentary on page 107.
Primary cardiac synovial sarcomas are rare cardiac tumors, with only 60 reported cases in the literature. ${ }^{1}$ Surgical resection is mandatory, but to date only the traditional approach though a median sternotomy has been described. This case report details a robotic, minimally invasive excision of a primary cardiac synovial sarcoma.

\section{CLINICAL SUMMARY}

The patient is a 19-year-old man who presented with a 1-week history of vague chest pain, episodic lightheadedness, and a systolic ejection murmur. An echocardiogram showed a well-circumscribed $6.6 \times 3.0-\mathrm{cm}$ left ventricular mass, with multiple calcifications and evidence of necrosis. The mass was attached to the posteromedial papillary muscle and exhibited movement into the left ventricular outflow tract (Figure 1, A, and Video 1). The maximal gradient was $22 \mathrm{~mm} \mathrm{Hg}$, and the ejection fraction was normal. Given these findings and the symptomatic nature of the lesion, the patient elected to undergo a minimally invasive robotic resection.

After induction of general anesthesia with a doublelumen endotracheal tube, a right groin cutdown was performed, and the femoral vessels were cannulated with a $20 \mathrm{~F}$ arterial cannula and $25 \mathrm{~F}$ long venous cannula. A right minithoracotomy in the 4th intercostal space was then performed, and the pericardium was incised and tacked to the lateral thoracic wall. Cardiopulmonary bypass with moderate hypothermia was used after adequate activated clotting time was achieved. The robotic arm trocars were introduced through separate stab incisions in the 3rd, 5th, and 6th intercostal spaces, and the camera trocar was situated within the right minithoracotomy. The robot (DaVinci $\mathrm{Xi}$; Intuitive Surgical, Sunnyvale, Calif) was then docked. The heart was arrested by crossclamping the aorta with a Chitwood clamp and infusing antegrade del Nido cardioplegia directly into the aortic root through a long aortic root vent.

A left atriotomy was made, and a dynamic mitral retractor was inserted deep into the left ventricle, providing excellent exposure. The tumor was noted to occupy most of the left ventricular cavity (Figure 1, $B$, and Video 1). The origin of the tumor was confirmed to be at the level of the posteromedial papillary muscle. The insertion of the tumor into the muscle was transected, and a figure-of-eight suture was placed at the base of the tumor for extraction. With 


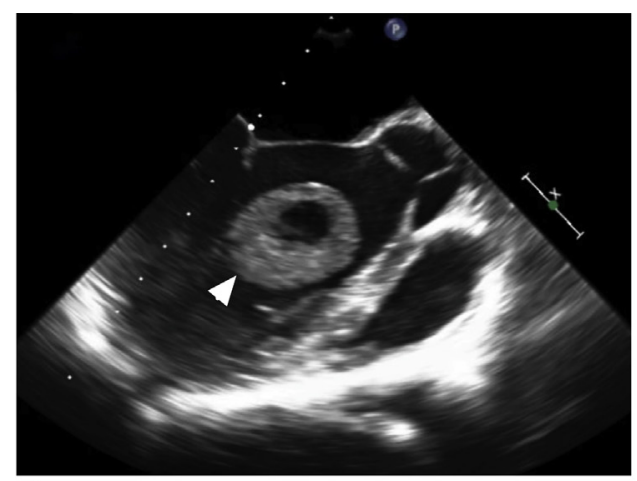

A

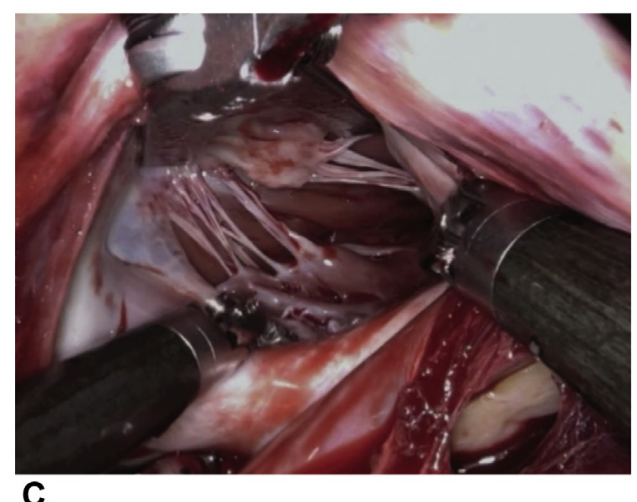

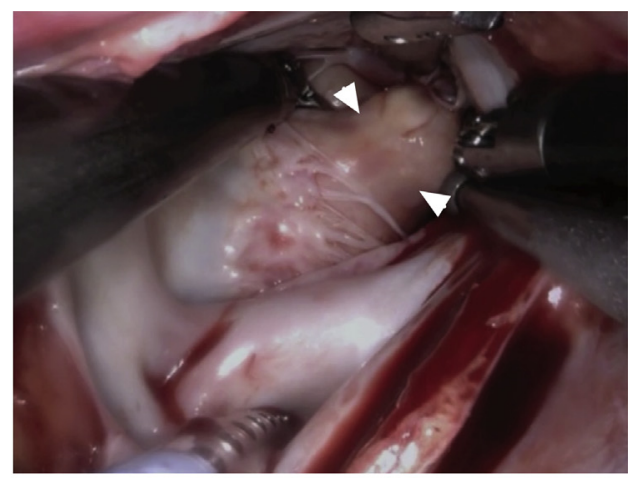

B

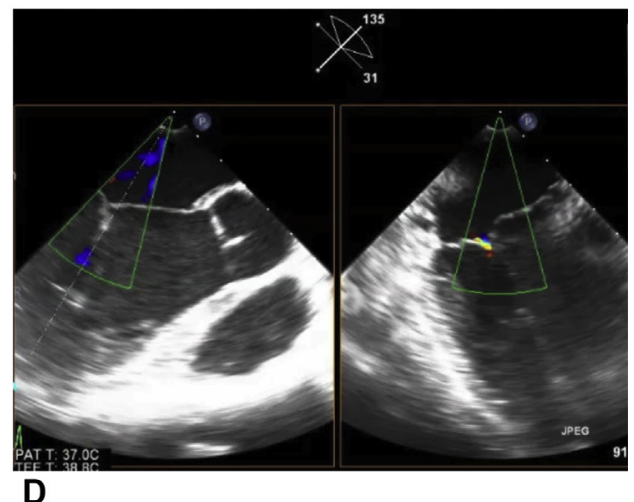

FIGURE 1. Perioperative Echocardiographic and Intraoperative Images. A, Preoperative echocardiogram showing a mobile 6.6 cm tumor (arrow) with movement toward the left ventricular outflow tract. B, Intraoperative view of the tumor (arrows) through the mitral valve. C, Intraoperative view of the left ventricle following tumor extraction. D, Post-operative echocardiogram showing no residual disease, trace mitral regurgitation.

gentle traction, the tumor was delivered into the left atrium and out of the heart with an endoscopic bag (EndoCatch; Medtronic, Minneapolis, Minn). The mitral valve and ventricle were carefully inspected; a small flail chorda tendinea was identified and repaired with a plication suture

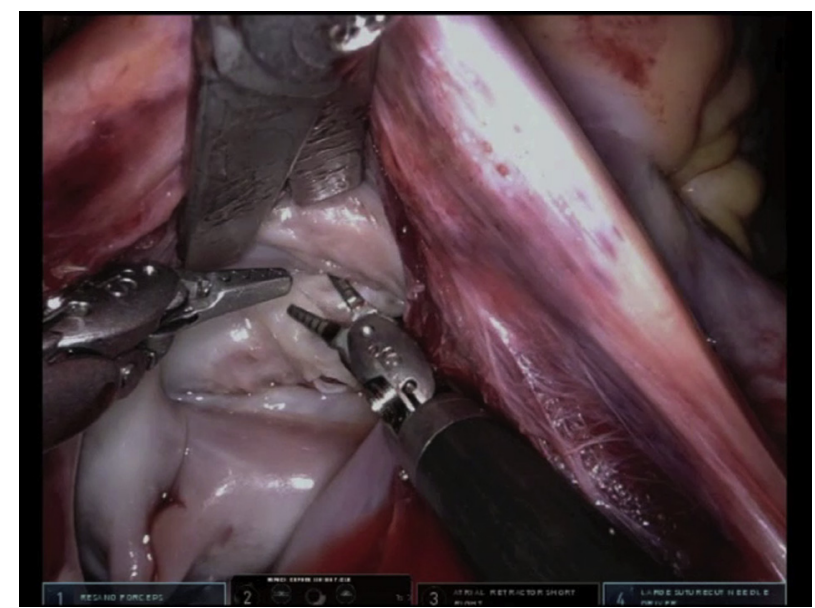

VIDEO 1. Preoperative echocardiogram showed a large $6.6 \mathrm{~cm}$ tumor in the left ventricle, with movement into the left ventricular outflow tract. Robotic resection was achieved via a left atriotomy. Video available at: https:// www.jtcvs.org/article/S2666-2507(20)30076-6/fulltext.
(Figure 1, $C$, Video 1). The atriotomy was closed, and the patient was weaned from cardiopulmonary bypass without inotropic support. Intraoperative transesophageal echocardiogram demonstrated normal ventricular function, trace mitral regurgitation, and no evidence of residual tumor (Figure 1, D, and Video 1). The total cardiopulmonary bypass time was 82 minutes.

The patient was transported to the intensive care unit and extubated 3 hours later. He was discharged on the fourth postoperative day, after an uneventful recovery. The final pathologic examination revealed a poorly differentiated, high-grade synovial sarcoma (Figure 2, $A$ and $B$ ). Fluorescence in situ hybridization was notable for $S Y T$ gene rearrangement, a characteristic feature of synovial sarcomas. The patient subsequently completed 6 cycles of adjuvant chemotherapy with doxorubicin and ifosfamide. At a 15-month follow-up, there was no evidence of recurrent disease and only trace physiologic mitral regurgitation (Figure 2, C). The patient provided informed consent for the publication of the study data.

\section{DISCUSSION}

Approximately $80 \%$ of primary cardiac tumors are benign and $20 \%$ malignant. Sarcomas are the most common malignant cardiac tumors, but synovial sarcomas 

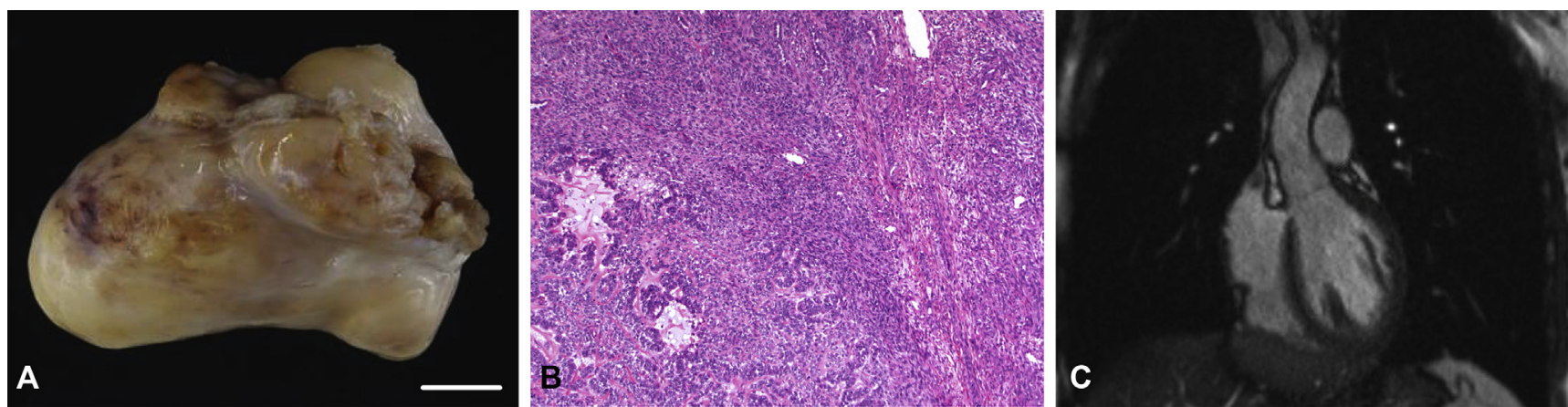

FIGURE 2. Pathological Examination and Follow-Up Magnetic Resonance Imaging. A, Gross pathology of the cardiac synovial sarcoma (scale bar, $1 \mathrm{~cm}$ ). $\mathrm{B}$, Hematoxylin and eosin histology of the lesion. C, Magnetic resonance imaging of the heart after 15 months, showing no recurrent disease.

account for only $4.2 \%$ of these. There are only 54 published articles describing cardiac synovial sarcomas, with a total of 60 patients (mean age, 37.1 years; male, $75 \%$ ). The median survival is approximately 24 months, and 1-year and 5-year survivals are $59.9 \%$ and $29.9 \%$, respectively, with chemotherapy and age significantly affecting overall survival. ${ }^{1}$ Surgical resection is indispensable, but only traditional approaches through a median sternotomy have been described.

There is limited experience with robotic resection of cardiac neoplasms, and most applications have been confined to benign lesions, namely atrial myxomas and papillary fibroelastomas. ${ }^{2-4} \mathrm{~A}$ single case involving resection of a primary cardiac lymphoma has been described. ${ }^{5}$ For cardiac tumor resections, robotic technology offers superior visualization, shorter lengths of stay, and higher quality of life scores. ${ }^{2,4}$ Our case suggests that oncologic outcomes are preserved as well, with no recurrence of disease after 15 months. This case thus demonstrates the feasibility of a minimally invasive robotic resection for rare malignant cardiac tumors.

\section{References}

1. Wang JG, Li NN. Primary cardiac synovial sarcoma. Ann Thorac Surg. 2013;95: 2202-9.

2. Yang M, Yao M, Wang G, Xiao C, Wu Y, Zhang H, et al. Comparison of postoperative quality of life for patients who undergo atrial myxoma excision with robotically assisted versus conventional surgery. J Thorac Cardiovasc Surg. 2015;150: 152-7.

3. Arsalan M, Smith RL, Squiers JJ, Wang A, DiMaio JM, Mack MJ. Robotic excision of a papillary fibroelastoma of the mitral chordae. Ann Thorac Surg. 2016; 101:e187-8.

4. Gao C, Yang M, Wang G, Wang J, Xiao C, Wu Y, et al. Excision of atrial myxoma using robotic technology. J Thorac Cardiovasc Surg. 2010;139:1282-5.

5. Moss E, Goldstein DA, Bradley KT, Flowers CR, Murphy DA. Successful robotic excision and early chemotherapy for primary cardiac lymphoma. Ann Thorac Surg. 2016;102:304-5. 